\title{
Cholangiocarcinoma metastasis to the esophagus
}

A 62-year-old man with stage IV cholangiocarcinoma presented with melena. An endoscopic ultrasound with fine-needle aspiration (FNA) of a large, multilobulated liver lesion 4 months earlier showed the cholangiocarcinoma. An endoscopic retrograde cholangiopancreatography (ERCP) was carried out to relieve left hepatic and common bile duct obstruction. The patient subsequently received carboplatin and gemcitabine, and presented with pancytopenia and a hematocrit of $20 \%$. Esophagogastroduodenoscopy (EGD) showed multiple pedunculated and ulcerated masses in the mid and distal esophagus ( $\bullet$ Fig. 1 , - Video 1). Biopsies revealed similar cells to those found on the prior FNA ( $\bullet$ Fig. 2). There were no further episodes of bleeding, and the patient has had no dysphagia or bleeding 4 months since discharge. The chemotherapy was changed to capecitabine and oxaliplatin. Surgery is reported to be successful and recommended for metastatic lesions with slow growing primaries [1].

This is the first report of a cholangiocarcinoma metastasizing to the esophagus. Esophageal metastases are rare but have been described from lung, breast, ovaries, and other primaries [1,2]. The typical endoscopic appearance of a metastatic esophageal lesion is a submucosal mass, although protruding as well as ulcerative lesions have been reported [1].

The pathophysiology includes hematogenous or lymphatic spread. The lymphatic drainage of the esophagus is unique and may explain our observations. The esophagus has lymphatics that extend into the lamina propria, as opposed to stopping at the submucosa, which is characteristic of the rest of the gastrointestinal tract. These lymphatic channels drain into the thoracic duct, which may, under conditions of altered or obstructed flow, act as a portal

\section{Video 1}

Esophagogastroduodenoscopy (EGD) of the mid and distal esophagus demonstrating pedunculated and ulcerated masses.

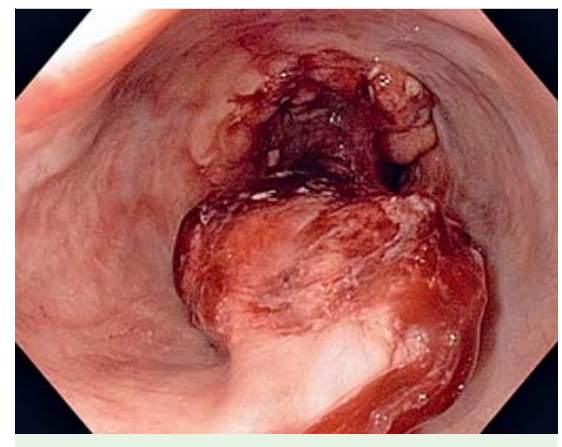

Fig. 1 Esophagogastroduodenoscopy (EGD) revealing pedunculated and ulcerated masses in the mid and distal esophagus.

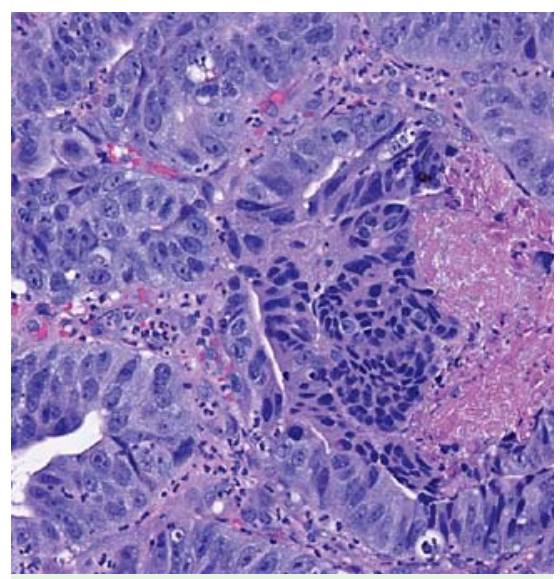

Fig. 2 Histopathological section shows invasive adenocarcinoma, moderately differentiated and similar in morphology to perigastric lymph node biopsies from previous endoscopic ultrasound/fine-needle aspiration (positive staining for CK7, CK20, and CDX2 suggestive of upper gastrointestinal tract origina, and negative for HepPar1 making liver origin less likely).

for metastasis from distant primaries. In fact, Zeidman showed that injecting tumor cells directly into the thoracic duct can lead to seeding of tissues, suggesting retrograde passage through efferent lymphatics [3]. It is important to keep a high index of suspicion for esophageal metastasis in patients with an existing cancer and new esophageal lesions.

\section{Competing interests: None}

Endoscopy_UCTN_Code_CCL_1AB_2AC_3AB
T. Sato, M. Krier, T. Kaltenbach,

\section{R. Soetikno}

Gastrointestinal Endoscopy Unit, Veterans Affairs Palo Alto Health Care System, Palo Alto, California, USA

\section{References}

1 Mizobuchi S, Tachimori Y, Kato H et al. Metastatic esophageal tumors from distant primary lesions: report of three esophagectomies and study of 1835 autopsy cases. Jpn J Clin Oncol 1997; 27: 410-414

2 Kagaya H, Kitayama J, Hidemura A et al. Metastatic esophageal tumor from cecal carcinoma. Jpn J Clin Oncol 2007; 37: 628 - 631

3 Zeidman I. Experimental studies on the spread of cancer in the lymphatic system. III. Tumor emboli in thoracic duct; the pathogenesis of Virchow's node. Cancer Res 1955; 15: 719-721

\section{Bibliography}

DOI $10.1055 / \mathrm{s}-0030-1255641$

Endoscopy 2010; 42: E250

(c) Georg Thieme Verlag KG Stuttgart - New York . ISSN 0013-726X

Corresponding author

R. Soetikno, MD

Gastrointestinal Endoscopy Unit, Veterans Affairs Palo Alto Health Care System Palo Alto, California,

USA

soetikno@earthlink.net 